\title{
¿El poder de mercado está asociado a variaciones positivas de precios en estructuras oligopólicas? El caso de las marcas líderes por sus ventas en la industria automotriz mexicana (2018)
}

\section{Eric Israel Ríos Nequis* Daniela Edith Carbajal Cansino** y Michelle Edith HeRnández Solís**}

\section{RESUMEN}

El artículo aborda las características de la industria automotriz, su estructura y concentración en distintos rubros o temáticas. La información recabada indica que es un oligopolio con medianos niveles de concentración, aunque posee un patrón de competencia distintivo: la diferenciación de productos, el cual explica la variabilidad de los precios en este sector industrial en tanto reduce la capacidad del poder del monopolio para incidir positivamente en ellos; se asume que el mercado es grande y capaz de soportar los niveles de diferenciación de producto y de mostrar poca sensibilidad a los cambios de precios.

Palabras Clave: Estructura de mercado, precios, oligopolio, organización industrial, automóviles.

Clasificación JEL: D43, D49, L10, L00, L62.

* Profesor-Investigador de Tiempo Completo. Universidad Autónoma de Baja California. Facultad de Ciencias Administrativas, Baja California, México. Correo electrónico: erios1uabc@ gmail.com

** Estudiantes del programa educativo de Comercio Internacional y Aduanas. Universidad Politécnica Metropolitana de Hidalgo, Hidalgo, México. Correo electrónico: decansino@outlook. com y solismichelle350@gmail.com, respectivamente 


\section{ABSTRACT}

Is market power associated with positive price variations in oligopolistic structures?: The case of the leading brands on sales in the Mexican automotive industry (2018)

This article deals with the characteristics of the automotive industry, its structure and concentration in different areas or topics. The information collected indicates that this industry is an oligopoly with medium concentration levels, although it has a pattern of distinctive competition: product differentiation, which explains the price variability in this industrial sector because reduces the capacity of the monopoly power to influence on the prices. It is assumed that the market is large and capable of supporting the levels of product differentiation and of showing little sensitivity to changes in the prices.

Keywords: Market Structure, Prices, Oligopoly, Industrial Organization, Automotive Industry

JEL Classification: D43, D49, L10, L00, L62.

\section{INTRODUCCIÓN}

Ante un nuevo contexto de apertura comercial, los ciclos productivos, las cadenas globales de valor y la entrada de competencia, se han experimentado cambios sustanciales en la estructura de mercado de la industria automotriz mexicana. En 1965 había sólo seis fabricantes de autos: los estadounidenses Chrysler, Ford, General Motors y Vehículos Automotores de México (con 40\% de la estructura del capital en manos del país del norte, mientras que el restante $60 \%$ era del gobierno mexicano); más la japonesa Nissan y la alemana Volkswagen (Solís, 2012).

La teoría clásica del comercio internacional indica que el libre comercio fomenta la entrada de nuevos competidores, productos y marcas, lo que brinda más opciones al consumidor y la posibilidad de comprar a precios económicos, bajo el supuesto de la entrada de competidores con ventajas absolutas, comparativas y otros factores que les permiten ser más eficientes, a través de la introducción de productos con precios accesibles. Asimismo, la síntesis poskeynesiana y neoclásica contempla la posibilidad de un comportamiento de precios distinto a los originados en la competencia, perfecta e imperfecta, como 
el monopolio, el oligopolio, la alineación, o en deflación, mediante el índice de alineación de precios (Anaya, 2008; 2012; 2014).

Steindl (1952) clasifica las estructuras de mercado, dentro de un contexto de apertura comercial, en industrias competitivas y en oligopolios competitivos, concentrados, diferenciados y diferenciados con altos niveles de concentración. En México existe evidencia empírica de la presencia de un oligopolio diferenciado y concentrado en la industria automotriz mexicana, mediante el índice de alineación de precios, con tendencia a disminuir (Anaya, 2008).

El análisis de precios constituye un tema de debate porque analiza desde el enfoque de la oferta para explicar su variabilidad en función de los factores de producción (Tansey y Raju, 2017), de modo que se acota la asociación entre el poder de mercado y el comportamiento de precios monopólicos con ciertos parámetros (Tansey y Raju, 2017). Sin embargo, algunos analistas abordan la sensibilidad de la demanda, la posibilidad de compra, los tipos de producto, los segmentos de mercado, el comportamiento de precios y el poder de mercado. Investigaciones previas muestra ciertas asociaciones entre poder de mercado y mayores márgenes de ganancias a través del precio bajo ciertas condiciones, como la utilidad de la demanda, el tipo de producto, los costos de fabricación y el tipo de comportamiento estratégico de las empresas en los distintos tipos de productos ofertados (Sudhir, 2001).

El objetivo principal del trabajo consiste en evaluar los elementos distintivos de producto asociados con la variabilidad de los precios finales en los consumidores, para el caso mexicano, en enero y febrero de 2018; se busca, particularmente, examinar la correlación positiva entre cuota de mercado y precios en México. Pretende demostrar que la estructura de mercado en la industria automotriz obedece a un oligopolio, según la teoría poskeynesiana, con diferenciación de producto y medianos niveles de concentración; sin embargo, el poder de mercado no resulta capaz de influir positiva ni significativamente en los precios porque la diferenciación es el elemento central de variación positiva, debido a las categorías basadas en los segmentos de mercado y nichos donde los ingresos y preferencias son plurales; por ende, los precios de las categorías automotrices se traducen en la misma forma, es decir, la dominancia de una empresa no necesariamente implicará liderazgo generalizado en precios, como establece la teoría ortodoxa de oligopolios. 
El trabajo de investigación comprende cuatro secciones fundamentales: la primera incluye el contexto teórico; la segunda integra un breve análisis de la concentración de la industria; luego, se aborda la metodología empleada y resultados econométricos; finalmente, se refieren discusión, conclusiones y limitaciones del estudio.

\section{Contexto teórico}

La teoría clásica ofrece una gama de alternativas para comprender la asociación entre poder de mercado e incremento de precios en las estructuras de mercado monopólicas, oligopólicas y monopolísticas. A medida que el poder de mercado aumenta, hipotéticamente existe mayor capacidad de afectar precios para obtener beneficios económicos superiores.

\subsection{La empresa dominante y sus implicaciones en el precio}

La literatura clásica sobre los juegos de oligopolio asume un patrón de competencia tradicional potencialmente válida en diferentes contextos y casos; el primer comportamiento es el modelo de la empresa dominante, relacionado positivamente con el poder de mercado. Analistas de la Teoría de la Organización Industrial, como Tarziján y Paredes (2006), mencionan que la empresa dominante enfrenta una curva de demanda con pendiente negativa y coexiste con una o más empresas pequeñas (seguidoras) que participan en el mismo mercado, circunstancia que las define.

Para los autores, el modelo de la empresa dominante supone que las menores poseen una menor influencia en la determinación de alguna variable, como precio o publicidad, ya que no pueden, mediante su acción individual, afectar las ventas del producto (Tarziján y Paredes, 2006).

Una de las críticas al modelo recae sobre los indicadores porque el poder de mercado no es la única vía para demostrar liderazgo. Para los teóricos, esta determinación debería depender también de la capacidad para ser la líder en precios, además de la conveniencia de contemplar información adicional, como tecnología y redes de distribución (Tarziján y Paredes, 2006).

La Figura 1 muestra de manera gráfica cómo la empresa dominante establece el precio por cobrar. La línea D representa la demanda de 
mercado y $\mathrm{S}_{\mathrm{p}}$, la oferta agregada de las empresas más pequeñas; así, la dominante precisa la demanda que enfrenta ella misma $\left(D_{d}\right)$ : como $S_{p}$ es la oferta de las empresas menores, $D_{d}$ manifiesta la diferencia entre la curva de demanda de mercado y la de oferta de las seguidoras.

Como ejemplo de la forma de derivar la demanda que afronta la empresa dominante, se considera que, al precio $\mathrm{P}_{1}$, la oferta de las empresas seguidoras es igual a la demanda de mercado, con lo que la empresa dominante no podría vender nada; en otras palabras, la demanda es cero. A un precio igual o menor que $\mathrm{P}_{2}$, las pequeñas no están dispuestas a vender el producto; por tanto, la empresa dominante cubre toda la demanda, mientras que, a precios entre $\mathrm{P}_{2}$ y $\mathrm{P}_{1}$, ésta enfrenta la curva $D_{d}$ de demanda.

A partir de la demanda $D_{d}$, la empresa dominante obtiene su ingreso marginal, que con el objetivo de maximizar sus utilidades, iguala con el costo marginal $\mathrm{CMg}_{\mathrm{Dd}}$. Al igualar vende una cantidad igual a $\mathrm{Q}_{\mathrm{d}}$ a un precio de $\mathrm{P}^{*}$. A este precio, las empresas pequeñas o seguidoras ofrecen una cantidad igual a $Q_{\mathrm{f}}$, de modo que la cantidad total vendida será igual a $Q_{d}+Q_{f}$.

Figura 1

COMPORTAMIENTO TEÓRICO DEL MODELO DE EMPRESA DOMINANTE

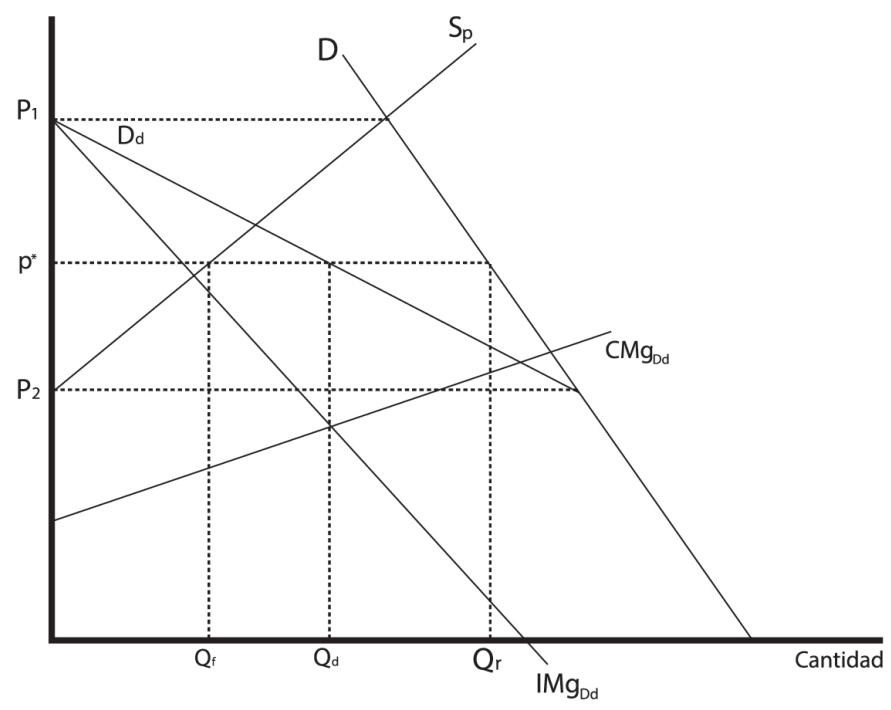

Fuente: Tarziján y Paredes, 2006. 
Para Tarziján y Paredes (2006), las empresas seguidoras actúan como competidoras al tomar como dado el precio establecido por la empresa dominante y producir la cantidad óptima a ese precio. En este modelo también existe algún grado de interdependencia estratégica, ya que la dominante establece su precio considerando cómo afectará la producción de las empresas más pequeñas. Entonces, ya que ambas asumen su tamaño como establecido, no lo determinan estratégicamente.

\section{EL PODER DE MERCADO Y SUS ASOCIACIONES A LA CONCENTRACIÓN Y PRECIOS}

El poder de mercado (Pm) es traducido como la capacidad de fijar precios; algunos economistas lo denominan poder de monopolio (Carlton y Perloff, 1994). Una situación como $\mathrm{p}>\mathrm{Cm}_{\mathrm{g}}$ implica menor producción y demanda con respecto al nivel de equilibrio, $\mathrm{p}=\mathrm{Cm}_{\mathrm{g}}$, con la afectación negativa del excedente del consumidor y del productor, así como de la eficiencia económica general (Anaya, 2012).

El $P m$ tiende a relacionarse directamente con la concentración industrial (ci), dado que entre mayor sea la $c i$, aumenta la capacidad de las empresas de afectar los precios positivamente, se logran acuerdos (explícitos o tácitos) y se restringe la cantidad de satisfactores que llegan al mercado (Anaya, 2012). Aunque puede haber excepciones, como los mercados impugnables con bajas barreras a la entrada, donde las estrategias competitivas de las empresas conllevan políticas de precios cercanos a los de la competencia y otras medidas (como un notorio exceso de capacidad instalada), a fin de limitar a los competidores y defender sus utilidades (П), situación estudiada por Brown y Domínguez (2005).

Las generalidades indican que la $c i$ se relaciona positivamente con $\Pi$ y, en consecuencia, con el poder de monopolio $P m$ de tal manera que, conforme aumenta una de las variables mencionadas, lo hacen las otras y viceversa, es decir, hay una relación bidireccional. En la literatura se ubican distintos analistas teóricos y empíricos que trabajan modelos y análisis económicos respecto al tamaño y distribución de П (Cabral, 1997; Church y Ware, 2000; Fernández de Castro y Duch, 2003).

En la Figura 2 se ilustra la asociación de estructuras de mercado con niveles diferenciados de $\Pi$, por un lado; por el otro, los niveles 
crecientes de $c i$ medidos en el índice de concentración absoluta $\mathrm{C}_{\mathrm{r} 4}$. La

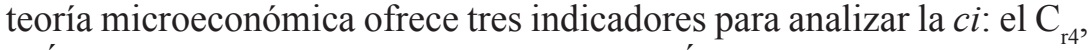
el Índice Herfindahl-Hirschman (IHH) y el Índice de Lerner; como se ha planteado, se vinculan con el poder de mercado.

El Cr4 y el Índice IHH son aplicados en evaluaciones econométricas de estructura-conducta y desempeño (ECD) para obtener conclusiones sobre la relación directa que suele suscitarse entre $P m$ y $c i$, así como sus efectos en las utilidades (Church y Ware, 2000); sin embargo, otros consideran que no necesariamente el poder del monopolio se manifiesta por ganancias mayores (Anaya, 2012). Actualmente los índices de concentración se aplican para resolver casos de competencia económica (prácticas monopólicas, por ejemplo), donde se busca disolver aglomeraciones industriales o el impedimento de fusiones que conllevan un aumento en la $c i$ (Utton, 1975; Cabral, 1997; Núñez, 2003). No obstante, una alta $c i$, aunque aporta información relevante, no es exclusiva del poder de mercado; resulta un indicador para analizar el poder de mercado y la prevención de sus consecuencias; ayuda, además, a identificar las estructuras de mercado y co-ayudan a describir el desempeño de la industria donde participan las empresas analizadas (Anaya, 2012).

Figura 2

PODER DE MERCADO Y CONCENTRACIÓN INDUSTRIAL

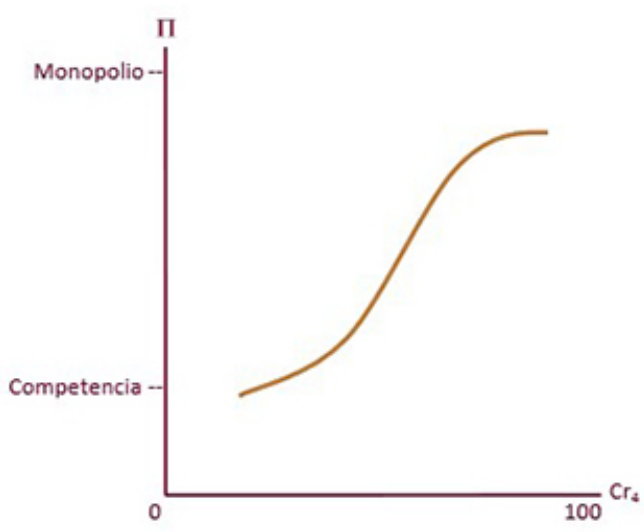


Por otro lado, el Índice de Lerner toma como referencia las características de la demanda; Lerner (1934) lo propone a fin de ordenar la magnitud del poder de mercado. En contraste con los índices de concentración $\mathrm{Cr} 4$ e IHH, los cuales toman como referencia la П, el de Lerner evalúa el margen de ganancia: analiza la $\Pi$ de manera directa (Anaya, 2012), donde su valor oscila entre 0 (escaso poder de mercado o monopolio) y 1 (caso extremo de poder de monopolio). El Índice de Lerner contempla la elasticidad de la demanda $\mathrm{e}_{\mathrm{d}}$ como herramienta de trabajo mediante la siguiente expresión:

$$
L=\frac{p-C m_{g}}{p}=-1 / e_{d}
$$

Donde $p$ es el precio y $\mathrm{C}_{\mathrm{mg}}$, el costo marginal; en otras palabras, se obtiene la diferencia del precio menos el costo marginal, o simplemente se aplica la inversa de la elasticidad de la demanda $\mathrm{e}_{\mathrm{d}}$. No obstante, el $\mathrm{C}_{\mathrm{r} 4}$ constituye un indicador frecuentemente utilizado para analizar variaciones de precios en un esquema Cournot (Stigler, 1968) y desde una perspectiva de alineación de precios para autos en México (Anaya y Esquivel, 2015), aplicado como una de las variables para evaluaciones longitudinales mayores a 40 años (Tansey y Rajú, 2017). Sin embargo, conviene acotar que el poder de mercado no necesariamente es el único indicador asociado a variaciones positivas de precios, porque implicaría omitir factores administrativos o de eficiencia como costos, salarios, margen de ganancia o elementos de competitividad como la diferenciación de productos.

\subsection{Estudios sobre la industria automotriz, poder de monopolio, patrones de competencia y análisis de precios}

La industria automotriz ha sido objeto de estudio desde diversos enfoques. Se ha evaluado el comportamiento de precios en contextos de competencia imperfecta: Lanzillotti (1958) detalló la forma en que General Motors cambiaba la tasa de beneficios y el margen de contribución con la finalidad de ganar participación de mercado; Halberstam (1986) describió los cambios en precios (reducción) de Ford durante la década de los cincuenta para acrecentar sus participaciones de mercado.

En Estados Unidos fue evaluada la colusión de precios como comportamiento típico de estructuras oligopólicas, donde las tres 
empresas más importantes de aquella época (Chrysler, Ford y General Motors) tendían a seguir el comportamiento de precios que una fijaba: si alguna reducía precios, las otras imitaban la estrategia; el resultado fue la ausencia de ganancias significativas en las participaciones de mercado y el sacrificio de márgenes de ganancia derivado de dichos movimientos estratégicos (Nag, 1983); tal fue un patrón común hasta finales de la década de los cincuenta, exceptuando la Segunda Guerra Mundial (Bresnahan, 1987).

El patrón de competencia cambió porque la estructura se comportó a partir de un modelo de liderazgo de precios donde General Motors los establecía secuencialmente y los seguidores (Ford y Chrysler) reaccionaban, tomándolos como referencia; en consecuencia, hubo precios homogéneos con leves diferencias entre ellos (Adams y Brook, 2005). Este paralelismo se tradujo en operaciones administrativas y de negocios, como la administración de personal, garantías y créditos financieros (Iacocca, 1986; Collier y Horowitz, 1987).

Los cambios en la estructura del mercado automotriz de Estados Unidos brindan la oportunidad de probar diversas hipótesis sobre la manera en que los precios responden a la concentración cambiante; para ello se ha empleado una amplia variedad de enfoques en el desarrollo de modelos. Levinsohn (1994) y Berry, Levinsohn y Pakes (1999) revisaron estudios que modelaron el mercado automotriz estadounidense y encontraron una significativa divergencia en lo que se trató como la variable dependiente; abarcan desde los márgenes brutos (Harless y Hoffer, 2002), los resultados (Takacs y Tanzer, 1986), los precios (Berry, Levinsohn y Pakes, 1999) e incluso la utilidad del consumidor y la elasticidad del consumo (West y Williams, 2005). Otros estudios longitudinales intentan probar que no hay cambios en las variaciones de participación de mercado y precios (Train y Winston, 2007).

Por otro lado, la variación de precios se explica por análisis de los factores de producción, mediante la asociación de los elementos que intervienen en la producción y generan variaciones en la dispersión de los precios (Berry, Levinsohn y Pakes, 1999; Tansey y Raju, 2017); en lo que concierne a la diferenciación de productos, Scherer (1980) demostró que los precios no presentaban cambios importantes ante sus homónimos en equipamiento y clasificación: eran semejantes por categoría. En un contexto más actual, se muestra la variabilidad de precios derivada de las preferencias de consumidor, traducidas en 
tipo de producto y marcas; destaca la mayor sensibilidad de los consumidores ante los precios de los autos estadounidenses que frente a los europeos y japoneses (Sudhir, 2001); sin embargo, se omiten variables que implican la estimación de alteraciones de precios y su vinculación con los tipos de productos y niveles de equipamiento.

\subsection{Estudios efectuados en México sobre industria automotriz}

En México se han realizado diversos trabajos para evaluar el contexto y la evolución de la industria automotriz, con la apertura comercial originada por el Tratado del Libre Comercio con América del Norte (TLCAN) como referencia, así como el desempeño de la industria con un enfoque microeconómico e indicadores específicos para compararla con sus homólogas respecto a las manufacturas, mediante artículos. Se tienen, además, memorias de congresos sobre la evolución de precios con aplicación de teoría económica heterodoxa.

Los trabajos empíricos evalúan los eslabones productivos de las cadenas de valor internacionales (Covarrubias, 2014), estudian su evolución tomando como variable estructural el TLCN (Ruiz, 2016) y analizan comparativamente los beneficios del modelo económico anterior y el actual en el ramo (Solís, 2012); también abordan el intercambio comercial con distintos mercados, como el brasileño, el estadounidense y el canadiense (Carbajal y Del Moral, 2014; Carbajal y Morales, 2016). En torno a las temáticas de comercio exterior, se ha examinado el intraindustrial, en el que el ramo automotriz constituye uno de los casos de análisis en las manufacturas (Sotomayor, 2008; López y Rodil, 2008; Quiroz, Munguía y Salgado, 2015; Lechuga y De la Cruz, 2017), mientras que en algunos otros constituye tema específico de análisis (Martínez y Álvarez, 2011).

Se ha tratado el tema de los precios y su evolución con un enfoque heterodoxo. La alineación de precios se ha evaluado en México porque se relaciona con el poder de mercado en cierta medida y bajo ciertas condiciones (Anaya, 2008; 2012; 2014); sin embargo, debe acotarse su aplicación a modelos nuevos: se ha demostrado que el Cr4 y el IHH son significativos en mayor medida que el Índice de Lerner sobre el cociente de alineación de precios (Anaya, 2016).

Como se aprecia, los estudios han prestado poca atención a los patrones de competencia sustentados en la diferenciación de productos 
y su efecto en los precios. La planeación y administración estratégica, apoyadas en el paradigma de la ECD, argumentan que la diferenciación como táctica permite obtener márgenes superiores de ganancias y evadir, en mayor medida, a la competencia, que una basada en eficiencia de costos con incidencia en reducciones de precios (Porter, 1985).

En una situación como la mencionada, se atribuye a dicha estrategia la posibilidad de generar variaciones que contrarresten el poder del monopolio y su relación con los precios; asimismo, se busca explicar que las necesidades de los clientes son cubiertas por la diferenciación y que el tamaño de mercado es lo suficientemente grande para soportar la segmentación, en tanto hay menos sensibilidad de los consumidores, tal como expresa el modelo teórico planteado en la próxima sección.

Conviene acotar que la evidencia empírica anterior se desarrolló con los índices de precios generalizados; este trabajo, por su parte, analiza precios finales para el consumidor de manera específica (modelo, categoría y nivel de equipamiento) y su vinculación con las estrategias comerciales y participaciones de mercado por modelo de autos.

\subsection{El modelo Stackelberg y la diferenciación de producto como explicación a la variación de precios}

El modelo de líder secuencial (Stackleberg) presenta similitudes con los de la empresa dominante; en esta teoría de oligopolios, se asume que la empresa líder tiene ciertas ventajas sobre las seguidoras y que muchas decisiones se toman secuencialmente: uno de los competidores decide qué hacer después de haber observado la resolución del otro, de manera que si una empresa es líder del mercado o se ha instalado antes que otra firma, está en posición de tomar decisiones con antelación (Tarziján y Paredes, 2006: 208).

Con el mismo ejemplo de competencia en cantidades analizado supra ("La empresa dominante y sus implicaciones en el precio"), se supone ahora que la firma 1 se mueve antes que la firma 2; ésta, cuando debe definir su nivel de producción, ya conoce la decisión tomada al respecto por la otra; entonces, la firma 1 asume que 2, cuando deba moverse, hará lo mejor posible para ella, tal como la 1 en la etapa anterior. La mejor suposición que hace la firma 1 es que la 2 se moverá a lo largo de su función de reacción (Tarziján y Paredes, 2006).

La evidencia empírica ofrece modelaciones teóricas para comprender por qué no necesariamente debe asociarse la concentración 
de mercado con variaciones de precios; la diferenciación de productos explica asociaciones positivas probablemente derivadas de la segmentación de mercados, así como los niveles de diferenciación, mediante las variaciones de equipamiento en los autos y sus clasificaciones de uso (SUV, lujo, compacto, subcompacto, deportivo, etcétera).

Una de las primeras aproximaciones teóricas de un modelo Stackelberg con diferenciación es el planteado por Dixit (1979). La modelación establece que un incremento en la diferenciación de producto generará un aumento en las utilidades de la empresa líder; la compañía evita una competencia férrea mediante tal estrategia, en consecuencia, alcanza mayores beneficios; si la empresa líder consigue un grado de diferenciación sumamente elevado, ocasionará una separación de los productos seguidores (dificultará la sustitución), lo que originaría ganancias en un escenario monopólico. El supuesto clave en este análisis es concebir que la entrada de competidores potenciales resulta exógena (Žigić, 2012).

Por otro lado, si se asume la existencia de una entrada endógena de competidores en la modelación Stackelberg, se tienen resultados distintos respecto al supuesto exógeno; en este caso, la ganancia del líder declina, mientras la diferenciación del producto aumenta, por ende, las utilidades se aproximan a cero en vez de ser monopolistas. El supuesto principal radica en que la demanda del tamaño de mercado no está asociada con aumentos en la entrada de competidores ni con el grado de diferenciación de productos (Žigić, 2012).

Para ilustrar la idea se consideran tres fases en un juego Stackelberg: en la primera, el líder entra, paga el costo $\mathrm{F}$ de ello y escoge el producto terminado que considera $\mathrm{qL}$; en la segunda, las empresas entrantes o seguidoras deciden ingresar y asumen los costos F; finalmente, éstas eligen un producto terminado para comercializar qi.

$$
u=U\left(q_{i} \ldots \ldots q_{m}\right)+q_{0}
$$

Como Dixit (1979), se asume que la demanda posee una función de utilidad cuasilinear:

Donde $\mathrm{q}_{0}$ sirve como función numeraria y de subutilidad; $\mathrm{U}\left(\mathrm{q}_{\mathrm{i}} \cdots\right.$ $\mathrm{q}_{\mathrm{m}}$ ) describe la utilidad que el consumidor deriva de las adquisiciones de productos diferenciados; la función es cuadrática y cóncava, como 
lo establece Dixit (1979). Asimismo, se plantea la existencia de una cantidad finita de consumidores, con ingresos finitos $\mathrm{Y}_{\mathrm{j}}<\infty$. Cada empresa enfrenta una demanda inversa para bienes diferenciados $i$ donde $\mathrm{P}_{\mathrm{i}}\left(\mathrm{q}_{\mathrm{i}} \sum j \neq \mathrm{i} \mathrm{q}_{\mathrm{j}}\right)=\mathrm{a}-\mathrm{q}_{\mathrm{i}}-b \sum j \neq \mathrm{i} \mathrm{q}_{\mathrm{j}}$; el parámetro $b \in(0,1)$ captura el grado de diferenciación de producto o sustitución alrededor de los productos. Por otro lado, las compañías deben pagar los costos fijos de inversión $\mathrm{F}>0$ para entrar e incurrir en una variable única de costo $\mathrm{c}>0$, la cual es constante.

\section{Problema del seguidor}

Primero se resuelve el problema del seguidor respecto a la solución final de producto terminado; se toman como referencia las cantidades de producto de la compañía dominante $\mathrm{q}_{\mathrm{L}} \mathrm{y}$ la cantidad de seguidores $\mathrm{m}$ que entraron al mercado, es decir, después de resolver $\mathrm{m}$ como una función de q_L; finalmente, la cantidad de entrantes se usa como reacción y $\mathrm{q}_{\mathrm{i}}$, como la condición del problema del líder. Se representa así:

$$
\operatorname{Max}_{q}\left\{\pi\left(q_{i}, q_{L}\right)\right\}=\max \left\{\left(P_{i}-C\right) q_{i}-F\right\}
$$

A partir de la condición de primer orden, cuando se resuelve el equilibrio simétrico entre seguidores, se obtiene:

$$
q_{i}\left(q_{L}, m\right)=\frac{\left(a-c-b * q_{L}\right)}{2+b(m-1)} .
$$

Ahora se encuentra la utilidad de cada seguidor; se obtiene por el número de seguidores como una función de la estrategia del líder $\mathrm{m}$ $\left(\mathrm{q}_{\mathrm{L}}\right)$ por utilizar la condición de utilidad cero; se expresa así:

$$
m\left(q_{L}\right)=\frac{a-c-(2-b) \sqrt{F}-b * q_{L}}{b \sqrt{F}}
$$

Sorpresivamente, la cantidad de competidores desciende con $\mathrm{q}_{\mathrm{L}}$, por ende, el líder actúa agresivamente y deja en el mercado la menor cantidad posible de espacios para la competencia (Žigić, 2012).

Destaca cómo la cantidad de producto final de cada seguidor cambia en el equilibrio con las cantidades de la empresa líder, a partir de dos efectos opuestos. La primera respuesta constituye el efecto directo $\partial q_{i} \frac{\left(m, A_{L}\right)}{\partial q_{L}}$, negativa desde el enfoque de la ecuación (1). El segundo, un efecto indirecto, se origina por un incremento en la cantidad de producto que genera un descenso en la cantidad de compañías seguidoras en el equilibrio, como se aprecia en la ecuación (2); por tanto, ejerce un 
efecto positivo en las cantidades de las empresas seguidoras desde

$\left(\frac{\partial q_{i}\left(m, q_{L} \nu\right.}{\partial m}\right)\left(\frac{d m}{d q_{L}}\right)>0$. Resulta que los dos efectos descritos se compensan y, en consecuencia, la acción de los seguidores no cambia con la táctica del líder.

A fin de comprobarlo se conecta la ecuación (1) con la (2) para obtener una respuesta en la estrategia de la empresa seguidora $\mathrm{q}_{\mathrm{i}}{ }_{\mathrm{L}}=\sqrt{\mathrm{F}}$; el precio resultante de un seguidor típico es $\mathrm{P}_{\mathrm{i}}=\mathrm{c}+\sqrt{\mathrm{F}}$. La estrategia de equilibrio de un seguidor no se ve afectada por la del líder cuando la entrada es libre, en lugar de que se establezca una amplia variedad de conductas de mercado (Etro, 2004 y 2008); por tanto, $\mathrm{q}_{\mathrm{L}}$ afectará el total de la cantidad de producto de las compañías seguidoras mediante $m$, no por $\mathrm{q}_{\mathrm{i}}$.

\section{El problema del líder}

Finalmente, el problema del líder es:

$$
\operatorname{Max}_{q i}\{\pi\}=\operatorname{Max}_{q i}\left\{\left(a-c-m * b * q-q_{L}\right) q_{L}-F\right\}
$$

Si se toman las condiciones de primer orden y se sustituyen (1) y (2), se obtiene:

$$
q_{L}=\frac{(2-b) \sqrt{ } F}{2(1-b)}>q_{i}
$$

El precio correspondiente resulta:

$$
P_{L}=c+\frac{1}{2}(2-b) \sqrt{F}<P_{i}
$$

Por tanto, el líder produce más que cada seguidor y carga un precio menor que la competencia. El equilibrio de utilidad del líder se expresa así:

$$
\pi_{L=} \frac{\left(b^{2} F\right)}{(4-4 b)}
$$

También se resuelve el problema del equilibrio, respecto a la cantidad de entrantes $\mathrm{m}$, conectando (3) en (2) para obtener:

$$
m^{*}=\frac{a-C}{b \sqrt{F}}+1-\frac{2-b}{2(1-b)}-\frac{2}{b}
$$

Como se ve inmediatamente en (4), el beneficio de acomodación del líder en la configuración aumenta en el parámetro de diferenciación b. Se intuye que, cuando los productos se parecen más (es decir, cuando 
aumenta b), la competencia se torna más intensa y, como consecuencia, menos firmas entran en equilibrio.

En otras palabras, el líder puede darse el lujo de sacar del mercado a más participantes potenciales a medida que los productos se vuelven menos diferenciados. Por tanto, aumentar la diferenciación del producto (dejar que b se mueva hacia cero) conduce de nuevo al resultado "no estándar" pero intuitivo; en este caso, el número de firmas que ingresan al mercado aumentaría y el beneficio del líder se iría hacia cero.

Se puede ver que, como se esperaba, el mayor grado de diferenciación del producto permite al líder cobrar precios más altos y generar menos en equilibrio. Como consecuencia, el precio del líder tiende al homólogo de un seguidor típico y su producción se destina a la salida de éste, ya que el grado de diferenciación del producto tiende a su límite. Por tanto:

$$
\lim _{b \rightarrow 0}\left\{c+\frac{1}{2}(2-b) \sqrt{F}\right\}=\lim _{b \rightarrow 0}\left\{a-b \cdot m^{*} \cdot q^{*}-q_{L}\right\}=c+\sqrt{F}
$$

Y

$$
\lim _{b \rightarrow 0}\left\{\frac{(2-b) \sqrt{F}}{2(1-b)}\right\}=\sqrt{F}
$$

Finalmente:

$$
\lim _{b \rightarrow 0}\left\{\pi_{L}\right\}=0
$$

El modelo asume que el líder no siempre manifiesta su liderazgo en precios por su posición competitiva, es decir, la posibilidad de que el poder de monopolio se traduzca en asociaciones positivas no resulta del todo convincente porque la diferenciación constituye el indicador que explica la variación de precios. De hecho, la variable mencionada se correlaciona con los competidores: a mayor cantidad de diferenciación de productos, mayor presencia de competidores. Esto tiene sentido con la industria automotriz en México porque, a causa de la apertura comercial, la cifra de competidores, modelos y marcas ha aumentado; se asume, entonces, la aparición de competencia exógena: la empresa dominante no necesariamente tiene control de quien ingresa o no, como se ejemplifica con la llegada de KIA y Hyundai al mercado nacional en 2014 y 2015, circunstancia que la líder (Nissan) no pudo impedir; lo mismo ocurrió con JAC.

Por otro lado, el modelo implica que el líder busca saturar la cantidad de espacios mediante diferenciación de productos; los segui- 
dores reaccionan compensando los efectos que ocasiona tal estrategia: los niveles de diferenciación llegan al límite donde el precio del líder difiere significativamente del fijado por un seguidor típico, en consecuencia, no caben los supuestos de la concentración ni del poder de monopolio ejercido mediante los precios. Estos escenarios son posibles siempre y cuando el mercado resulte lo suficientemente grande para comprar productos a distintos precios, resultado de la diferenciación de producto. Una de las limitantes del modelo radica en que no explica de forma precisa la segmentación de mercados, si bien estipula que el nivel de diferenciación reside en la diversidad de productos, lo cual implica llegar a distintos nichos.

La función de la propuesta econométrica es la siguiente:

$$
\text { Precio } f=\left(D_{P}, S_{M}, P_{M}\right)
$$

Donde:

$\mathrm{D}_{\mathrm{P}}=$ Diferenciación de producto

$\mathrm{S}_{\mathrm{M}}=$ Segmentación de mercados

$\mathrm{P}_{\mathrm{M}}=$ Poder de monopolio

\section{CONTEXTO COMPETITIVO DE LA INDUSTRIA AUTOMOTRIZ}

Como se ha mencionado anteriormente, la evidencia empírica demuestra que la industria automotriz es un caso de oligopolio diferenciado con un nivel de concentración moderadamente alto (Anaya, 2008), además, variable por criterio de análisis (producción, exportaciones, ventas y ventas por segmentos automotrices). Un aporte del trabajo reside en describir tales variaciones y otros datos destacados sobre el análisis de ventas y el comportamiento estratégico.

La Asociación Mexicana de la Industria Automotriz (AMIA) proporciona los datos mensuales de las ventas totales por comercializador o producto en el país. La Tabla 1 del anexo muestra que la industria no presenta altos niveles concentración, tanto el $\mathrm{C}_{\mathrm{r} 4} \mathrm{y}$ el Índice Herfindahl señalan que no hay presencia de dicho fenómeno (66\% y 1346, respectivamente); resulta menor en las ventas de camiones ligeros (un $\mathrm{C}_{\mathrm{r} 4}$ de $54 \%$ y un índice de 1141). Destaca que, después de la firma del TLCAN, la cantidad de competidores aumentó drásticamente de 6 a 24 participantes aproximadamente (ver tabla 2 del Anexo); sin embargo, la 
información de la AMIA (2018) señala la centralización en tres categorías (Tabla 2).

\subsection{Metodología}

El estudio es de corte cuantitativo y de alcance correlacional; el instrumento se basa en un modelo de regresión de corte transversal con 115 observaciones o versiones de automóviles de distintas marcas, agrupados en las categorías de auto subcompacto, compacto, sedán, SUV, sedán de lujo y deportivo. La variable dependiente es el precio (numérico, discontinuo y transformado a logaritmo), mientras las independientes son numéricas, discontinuas y convertidas en logaritmos (participación de mercado y cantidad de competidores); también se consideran otras independientes: cualitativas y ficticias, para determinar la versión de equipamiento (básico, intermedio o equipado), así como su clasificación (subcompacto, compacto, sedán, sedán de lujo, deportivo y SUV).

Las fuentes de información fueron la Asociación Nacional de Distribuidores Automotrices (AMDA) y las páginas en internet de las marcas de donde se obtuvieron las versiones para determinar las dummy y sus precios.

Las marcas involucradas de subcompactos, por categoría, fueron Vento, March, Aveo, Beat y Río; de compactos, Sentra, Versa, Jetta, Honda City y Mazda 3; de sedanes de lujo, Honda Accord, Toyota Prius, Toyota Camry, Nissan Altima y Volkswagen Passat; de deportivos, Seat León, Ford Mustang, Mazda MX5, Seat León SC y Chevrolet Camaro; finalmente, los de uso múltiple, Honda CR-V y HR-V, Nissan Kicks, Kia Sportage y Nissan X-trail.

La especificación de los modelos es la siguiente:

$$
\begin{aligned}
L n_{\text {precio }}=\beta_{0} & +\beta_{1 \text { ln }} \text { competidores }+\beta_{2 \text { tn }} \text { poder de mercado }+\beta_{3 \text { dum }} \text { subcompacto } \\
& +\beta_{4 \text { dum }} \text { compacto }+\beta_{\text {sdum de lujo }}+\beta_{6 \text { dum }} \text { deportivos }+\beta_{7 \text { dum }} \text { suv } \\
& +\beta_{\text {gdum }} \text { Equip. Básico }+\beta_{9 d u m} \text { Equip. Interm. }+\beta_{10 d u m} \text { Equip. Alto }+\varepsilon
\end{aligned}
$$

El modelo es una función log-log con competidores y poder de mercado, así como una función log-dummy con las restantes, para visualizar cómo se determinaron los efectos porcentuales y la descripción de las variables (ver cuadro 3 del Anexo). 


\subsection{Resultados}

Los modelos econométricos de corte transversal poseen coeficientes de determinación de 0.8165 y 0.8269 , es decir, $81.65 \%$ y $82.69 \%$ de la oscilación de los precios está correlacionada por las variables que integran las regresiones (ver Tabla 3). Se obtuvieron resultados significativos con $99 \%$ de confianza; las pruebas estadísticas de distribución normal de los errores, correcta especificación, varianza homogénea de los errores y colinealidad fueron satisfactorias (ver Pruebas de normalidad en el Anexo). Los efectos marginales indican que, al aumentar en $1 \%$ la cantidad de competidores, los precios de los automóviles estarán asociados a descensos de $0.2264 \%$ y $0.1889 \%$ en enero y febrero (ver Tabla 3).

El poder de mercado, por su parte, posee una asociación negativa con los precios; los efectos marginales señalan que, al aumentar en $1 \%$ la participación de mercado de las marcas, los precios estarán asociados a un declive de 0.1814 y $0.2289 \%$, lo cual contradice los resultados de Tansey y Raju (2017), Moraga, Sandor y Wildenbeest (2017), pero son congruentes con los hallazgos de Sudhir (2001), quien encuentra que, en segmentos donde hay tendencias negativas, las empresas aplican políticas de precios agresivas para contener o aumentar la participación del mercado. Este resultado coincide con lo encontrado por Lanzilloti (1958), Nag (1983) y Halberstam (1986), para quienes el aumento de participación se correlaciona negativamente con los precios, si bien los efectos negativos se muestran pequeños.

Las características de diferenciación de productos, por su categoría y segmentación de mercado (variables dummy), indican que los autos compactos poseen precios más altos en $36.70 \%$ y $37.49 \%$ respecto a los subcompactos; los autos de lujo, 75.77\% y 72.80\% más que los subcompactos; los deportivos, $125.37 \%$ y $127.65 \%$ más elevados que los subcompactos; los de uso múltiple, $72.03 \%$ y $71.52 \%$ altos que los subcompactos (ver Tabla 3). Los resultados son consistentes con Sudhir (2001), quien encuentra mayor dispersión y variaciones de precios entre los distintos segmentos; en su estudio encuentra diferencias de precios superiores en los autos compactos y medianos en Estados Unidos. Por otro lado, destaca que se confirman los resultados de Moraga, Sandor y Wildenbeest (2017). 
¿El poder de mercado está asociado a... $\Gamma$ Ríos N., E. I.; D. E. Carbajal C. y M. E. Hernández S.

Tabla 3

RESULTADOS DE LA ESTIMACIÓN ECONOMÉTRICA (ENERO Y FEBRERO DE 2018)

\begin{tabular}{ccccccccccc}
\hline $\begin{array}{c}\text { (Enero) } \mathrm{R}^{2} \\
\text { Variable }\end{array}$ & .8165 & Obs & 115 & Efec\% & (Feb.) R & .8269 & Obs & 115 & Efec\% \\
\hline $\begin{array}{c}\text { Competidores } \\
\text { Poder- }\end{array}$ & -.2264803 & -5.83 & 0.000 & -.2264 & Competidores & -.1889526 & -6.51 & 0.000 & -.1889 \\
\hline $\begin{array}{c}\text { mercado } \\
\text { Compacto }\end{array}$ & -.1814163 & -3.40 & 0.001 & -.1814 & $\begin{array}{c}\text { Poder- } \\
\text { mercado }\end{array}$ & -.2289722 & -3.60 & 0.000 & -.2289 \\
\hline Lujo & .583457 & 9.09 & 0.000 & 75.77 & Lujo & .5469865 & 9.03 & 0.000 & 72.80 \\
\hline Deportivo & .8280719 & 12.70 & 0.000 & 125.37 & Deportivo & .8226818 & 13.28 & 0.000 & 127.65 \\
\hline SUV & .5548221 & 11.13 & 0.000 & 72.03 & SUV & .5395495 & 11.40 & 0.000 & 71.52 \\
\hline Intermedio & .166367 & 4.83 & 0.000 & 18.17 & Intermedio & .1670865 & 4.97 & 0.000 & 18.18 \\
\hline Equipado & .2150743 & 4.25 & 0.000 & 24.10 & Equipado & .2221771 & 4.50 & 0.000 & 24.87 \\
\hline
\end{tabular}

Fuente: Elaboración propia con software Stata.

En lo que concierne a los niveles de equipamiento, éstos influyen en menor medida sobre los precios, si bien con efectos nada despreciables. Los resultados indican que los autos con equipamientos intermedios obtienen precios superiores en $18.17 \%$ y $18.18 \%$ por encima de las versiones básicas, mientras que aquellos con equipamientos más amplios fijan precios $24.10 \%$ y $24.87 \%$ más altos que las versiones austeras (ver Tabla 3). Los hallazgos son congruentes con Moraga, Sandor y Wildenbeest (2017) porque el equipamiento refleja efectos positivos en los precios; según los autores, la razón de las preferencias de los consumidores por artefactos como el control de crucero son un elemento atractivo dentro los niveles de equipamiento, respecto a las versiones que carecen de ello.

\section{CONCLUSIONES}

El trabajo demuestra, mediante patrones de competencia y precios (con base en la segmentación), que el poder de mercado no necesariamente debe traducir sus efectos en los precios de la industria automotriz mexicana porque la diferenciación de productos explica parcialmente sus fluctuaciones. Dentro de las estrategias empresariales más importantes se ubica el diseño de producto basado en las preferencias de los consumidores que, como puede apreciarse en los resultados, conlleva amplias variaciones; en menor medida se presentan los efectos del 
nivel de equipamiento potenciales de las versiones de cada vehículo, que influyen en la posibilidad de obtener el excedente del consumidor mediante aumentos de precios.

Conviene acotar la existencia de segmentos atractivos por su tamaño (ventas totales), y con mnores variaciones respecto a otros, tales son los casos de los autos compactos y de uso múltiple (SUV), los cuales son más grandes en ventas por volumen que los de lujo y los deportivos. Lo anterior, demuestra que la variación de precios es amplia, en tanto el tamaño de mercado lo permita, tal como lo plantea el modelo teórico referido: el líder adopta un precio similar al de un seguidor típico, así se cumple el límite de la diferenciación y se contrarresta el poder de mercado manifestado mediante precios, porque el liderazgo no se traduce necesariamente en precios.

Por otro parte, el supuesto de competencia imperfecta se cumple puesto que, como es conocido, en industrias oligopólicas las empresas competidoras enfrentan mayor dificultad para influir sobre los precios que los monopolios, a raíz de un menor control de costos. No obstante, su influencia puede desencadenarse por la diferenciación de producto: así se demuestra en una estructura oligopólica con mediana concentración, diferenciación alta y precios variables para los consumidores, lo que la aleja de un comportamiento alusivo a la guerra de precios.

\section{Biblografía}

AMDA (Asociación Mexicana de Distribuidores Automotrices) (2018), "Reportes", <https://www.amda.mx/> [marzo de 2018].

AMIA (Asociación Mexicana de la Industria Automotriz) (2018), en http:// www.amia.com.mx/ [marzo de 2018].

Adams, W. y Brook, J. (2005), The Structure of American Industry. Upper Saddle River, Prentice Hall, New Jersey.

Anaya, A. (2008), "El cociente de alineación de precios como indicador de poder de mercado", Economía Informa, 351, pp.113-125.

Anaya, A. (2012), "Poder de mercado. Nota teórica y referencias de su medición convencional con precios relativos", Ciencia Económica (1) 1, pp. 25-48.

Anaya, A. (2014), “Oligopolio y poder de mercado. Discusión de la hipótesis de precios rígidos", Economía Informa, 384, pp. 109-127.

Anaya, A. y Esquivel, M. (2015), “Automóviles nuevos en México: competencia y precios", Ponencia en el XXV Coloquio Mexicano de Economía 
Matemática y Econometría.

Berry, S.; Levinsohn, J. y Pakes, A. (1999), "Voluntary Export Restraints on Automobiles: Evaluating a Trade Policy", American Economic Review, 89 (3), pp. 400-430.

Bresnahan, Timothy (1987), "Competition and Collusion in the American Automobile Industry: The 1955 Price War", Journal of Industrial Economics, 35 (4), pp. 457-482.

Brown, F. y Domínguez, L. (2005), Organización industrial. Teoría y aplicaciones al caso mexicano, Facultad de Economía/UNAM, México.

Cabral, L. (1997), Economía industrial, McGraw-Hill, Madrid.

Carbajal, Y. y Del Moral, L. (2014), “El desempeño del sector automotriz en México en la era TLCAN. Un análisis a 20 años", Paradigma Económico, 6 (2), pp. 95-126.

Carbajal, Y. y Morales, M. (2016), “El sector automotriz en México y Brasil: un análisis desde la perspectiva comercial”, Revista Eletrônica de Negócios Internacionais, 11 (3), pp. 3-41.

Carlton, D. y Perloff, J. (1994), Modern Industrial Organization, Harper-Collins, Nueva York.

Church, J. y Ware, J. (2000), Industrial Organization. A Strategic Approach, McGraw Hill, Singapur.

Collier, P. y Horowitz, D. (1987), The Fords: An American Epic, Summit Books, Nueva York.

Covarrubias, A. (2014), "Explosión de la Industria Automotriz en México: De sus encadenamientos actuales a su potencial transformador”, Análisis, 1. Fundación Friedrich Ebert en México. pp.1-44. < http://library.fes.de/pdffiles/bueros/mexiko/10645.pdf >

Lechuga, J. y De la Cruz, C. (2017), “Comercio intra-industrial en las empresas manufactureras en México, a veinte años del inicio del TLCAN”, en Análisis Económico, 80 (32), pp. 46-68.

Dixit, A. (1979), “A Model of Duopoly Suggesting a Theory of Entry Barriers", en Bell Journal of Economics, 10 (1), pp. 20-32.

Etro, F. (2004), "Innovation by Leaders", The Economics Journal 114 (495), pp. 281-310.

Etro, F. (2008), "Stackelberg competition with endogenous entry", The Economics Journal, 118 (532), pp. 1670-1697.

Fernández de Castro, J. y Duch, N. (2003), Economía industrial. Un enfoque estratégico, McGraw-Hill, Ciudad de México, México.

Greene, W. (2019), Econometric Analysis, Pearson, Nueva York.

Halberstam, D. (1986), "The Reckoning”, Avon Books, Nueva York.

Harless, D. y Hoffer, G. (2002), "Do Women Pay More for New Vehicles? Evidence from Transaction Price Data", The American Economic Review, 92 (1), pp. 270-279. 
Iacocca, L. (1986), Iacocca: An Autobiography, Bantam Books, Toronto.

Levinsohn, J. (1994), "International Trade and the U.S. Automobile Industry: Current Research, Issues, and Questions", Japan and the World Economy, 6 (4), pp. 335-357.

Nag, A. (1983), "High New-Car Prices Keep Many Lookers Looking”, Wall Street Journal, 3 de agosto, Nueva York.

Núñez, F. (2003), El poder de mercado en la legislación de la competencia económica. Patria, México.

Moraga, J.; Sandor, Z. y Wildenbeest, R. (2017), "Consumer Search and Prices in the Automobile Market", IESE Business School Working Paper, 123E, Kelley School of Business Research Paper,15-45, <https://ssrn.com/ abstract $=2609338>$.

Lanzillotti, R. (1958), "Pricing Objectives in Large Companies", American Economic Review, 48 (5), pp. 921-940.

Lerner, A. (1934), "The concept of Monopoly and the measurement of Monopoly Power", Review of Economic Studies, 1 (3), pp. 157-175.

López, J. y Rodil, O. (2008), “Comercio intra-industrial e intra-firma en México en el contexto del proceso de integración de América del Norte (19932006)", Economía UNAM, 5 (13), pp. 86-112.

Martínez, A. y Álvarez, G. (2011), "Estructura de mercado y comercio intraindustrial en el sector automotriz", Revista de Estudios Económicos, 6 (12), pp.88-116.

Porter, M. E. (1985), The Competitive Advantage: Creating and Sustaining Superior Performance, Free Press, Nueva York.

Quiroz, S.; Munguía, G. y Salgado, M. (2015), "Comercio intra industrial México-América 2014-2015”, Economía Actual, 8, pp. 21-26.

Ruiz, C. (2016), "Desarrollo y estructura de la industria automotriz en México", Análisis, 6, pp. 1-32.

Scherer, F. M. (1980), Industrial Market Structure and Economic Performance, Rand Mc Nally, Chicago.

Solís, E. (2012), Apertura comercial de la Industria Automotriz en México: Un análisis de 1962 a 2009, Investigaciones Jurídicas de la UNAM, <https:// archivos.juridicas.unam.mx/www/bjv/libros/7/3021/12.pdf $>$ [26 de abril de 2018].

Sotomayor, M. (2008), Un estudio sobre el comercio-intra-industrial de México en el Tratado del Libre Comercio de América del Norte, tesis de Doctorado, Universidad Autónoma de Barcelona, Barcelona.

Steindl, J. (1952), Maturity and Stagnation by Mean of Commodities, Basil Blackwell, Oxford.

Stigler, G. (1968), The Organization of Industry, University of Chicago Press, Chicago.

Sudhir, K. (2001), "Competitive Pricing Behavior in the Auto Market: A Structural Analysis”, Marketing Science, 20 (1), pp. 42-60. 
Tansey, M. y Raju, S. (2017), "Pricing, concentration \& public policy: The U.S. automobile market", Journal of Policy Modeling, 39 (5), pp. 762-774.

Takacs, W. y Tanzer, E. (1986), "Structural Change in the Demand for Automobiles by Size Class", The Quarterly Review of Economics and Business, 26, pp. 48-57.

Tarziján, J. y Paredes, R. (2006), Organización industrial para la estrategia empresarial, Pearson-Prentice Hall, México.

Train, K. y Winston, C. (2007), "Vehicle Choice Behavior and the Declining Market Share of U.S. Automakers", International Economic Review, 48 (4), 1469-1496.

West, S. y Williams, R. (2005), "The Cost of Reducing Gasoline Consumption”, American Economic Review, 95 (2), pp. 294-299.

Utton, M. A. (1975), La concentración industrial, Alianza, Madrid.

Žigić, K. (2012), "Stackelberg leadership with product differentiation and endogenous entry: some comparative static and limiting results", Journal of Economics, 106 (3), pp. 221-232. 


\section{ANEXOS}

Regresión (enero 2018)

Homocedastcidad (Breusch-Pagan)

Breusch-Pagan / Cook-Weisberg test for heteroskedasticity

Ho: Constant variance

Variables: fitted values of Iprecio

$$
\operatorname{chi} 2(1)=1.50
$$

Prob $>$ chi2 $=0.2212$

Regresión (febrero 2018)

Breusch-Pagan / Cook-Weisberg test for heteroskedasticity

$$
\text { Ho: Constant variance }
$$

Variables: fitted values of Iprecio

$$
\operatorname{chi} 2(1)=2.85
$$

Prob $>$ chi2 $=0.0912$

Fuente: Elaboración propia con software Stata 12.

Enero (2018)

Test de Ramsey (especificación)

$F(3,103)$

Prob $>$ F

Prob $>$ F

\begin{tabular}{|c|c|c|c|c|c|}
\hline \multicolumn{6}{|c|}{ Skewness/Kurtosis tests for Normality } \\
\hline Variable & Obs & $\operatorname{Pr}($ Skewness) & $\operatorname{Pr}$ (Kurtosis) & adj $\mathrm{Chi}^{2}$ & Prob $>\mathrm{Chi}^{2}$ \\
\hline $\mathbf{r}$ & 115 & 0.0375 & 0.9018 & 4.45 & 0.1083 \\
\hline \multicolumn{6}{|c|}{ Febrero (2018) } \\
\hline \multicolumn{6}{|c|}{ Skewness/Kurtosis tests for Normality } \\
\hline Variable & Obs & $\operatorname{Pr}($ Skewness) & $\operatorname{Pr}$ (Kurtosis) & adj $\mathrm{Chi}^{2}$ & Prob $>\mathrm{Chi}^{2}$ \\
\hline $\mathbf{R}$ & 115 & 0.0307 & 0.3731 & 5.35 & 0.0688 \\
\hline
\end{tabular}

Fuente: Elaboración propia con software Stata 12

Enero (2018) Pruebas de normalidad

Fuente: Elaboración propia con software Stata 12.
2.25

0.0875

Febrero (2018)

1.7

0.1716 
PRUEBa DE COLINEALIDAD

\begin{tabular}{cccccc}
\hline \multicolumn{2}{c}{ Enero (2018) } & \multicolumn{2}{c}{ Febrero (2018) } \\
\hline VIF & VIF & 1/VIF & Variable & VIF & $1 /$ VIF \\
\hline Variable & 2.39 & 0.418567 & Deportivos & 2.38 & 0.418567 \\
\hline Deportivos & 1.87 & 0.534165 & De lujo & 1.9 & 0.534165 \\
\hline De lujo & 1.8 & 0.555303 & Iparticipacion & 1.8 & 0.555303 \\
\hline Iparticipacion & 1.73 & 0.577099 & Icompetidores & 1.73 & 0.577099 \\
\hline Icompetidores & 1.54 & 0.647814 & Múlltiples & 1.54 & 0.647814 \\
\hline Múlltiples & 1.53 & 0.654844 & Compacto & 1.53 & 0.654844 \\
\hline Compacto & 1.26 & 0.795962 & Equipado & 1.26 & 0.795962 \\
\hline Equipado & & & & & \\
\hline
\end{tabular}

Fuente: Elaboración propia con software Stata 12.

\section{Cálculo de los efectos porcentuales de una variable logarítmica}

Según Greene (2012), un modelo con variable dependiente logarítmica y regresores dummy se representa así:

$$
\ln Y=\beta 1+\beta 2 x+\beta 3 d+\varepsilon
$$

El coeficiente de la variable dummy $\mathbf{d}$ indica un cambio multiplicativo de la función. El porcentaje de cambio en $\mathrm{E}[\mathrm{y} \mid \mathrm{x}, \mathrm{d}=1]$, asociado a un cambio en $\mathbf{d}$, es:

$$
\begin{gathered}
\%\left(\frac{\Delta E[y \mid x, d]}{\Delta d}\right)=100 \%\left\{\frac{E[y \mid x, d=1]-E[y \mid x, d=0]}{E[y \mid x, d=0]}\right\} \\
=100 \%\left\{\frac{\exp (\beta 1+\beta 2 x+\beta 3 E[\exp (\varepsilon)]-\exp (\beta 1+\beta 2 x) E[\exp (\varepsilon)}{\exp (\beta 1+\beta 2 x) E[\exp (\varepsilon)]}\right\} \\
=100 \%[\exp (\beta 3)-1]
\end{gathered}
$$


Tabla 1

VENTAS Y CONCENTRACIÓN DE LA INDUSTRIA AUTOMOTRIZ EN CANTIDADES (FEBRERO DE 2018)

\begin{tabular}{|c|c|c|c|c|c|c|}
\hline & Autos & Cuota \% & $\mathrm{IHH}$ & Camiones ligeros & Cuota \% & $\mathrm{IHH}$ \\
\hline ACURA & 34.00 & 0.049 & 0.002 & 92.00 & 0.228 & 0.052 \\
\hline BMW & 1507.00 & 2.185 & 4.775 & 0.00 & 0.000 & 0.000 \\
\hline FCA MÉXICO & 3054.00 & 4.428 & 19.609 & 4031.00 & 9.975 & 99.496 \\
\hline FORD MOTOR & 2904.00 & 4.211 & 17.730 & 3176.00 & 7.859 & 61.765 \\
\hline GENERAL MOTORS & 11897.00 & 17.250 & 297.572 & 3473.00 & 8.594 & 73.857 \\
\hline HONDA & 2648.00 & 3.840 & 14.742 & 4443.00 & 10.994 & 120.874 \\
\hline HYUNDAI & 2397.00 & 3.476 & 12.080 & 1610.00 & 3.984 & 15.872 \\
\hline INFINITI & 79.00 & 0.115 & 0.013 & 120.00 & 0.297 & 0.088 \\
\hline ISUZU & 0.00 & 0.000 & 0.000 & 130.00 & 0.322 & 0.103 \\
\hline JAGUAR & 27.00 & 0.039 & 0.002 & 0.00 & 0.000 & 0.000 \\
\hline KIA & 5070.00 & 7.351 & 54.042 & 2538.00 & 6.280 & 39.442 \\
\hline LAND ROVER & 0.00 & 0.000 & 0.000 & 74.00 & 0.183 & 0.034 \\
\hline LINCOLN & 22.00 & 0.032 & 0.001 & 153.00 & 0.379 & 0.143 \\
\hline MAZDA & 2301.00 & 3.336 & 11.131 & 2052.00 & 5.078 & 25.783 \\
\hline MERC. BENZ & 1691.00 & 2.452 & 6.012 & 0.00 & 0.000 & 0.000 \\
\hline MINI & 523.00 & 0.758 & 0.575 & 0.00 & 0.000 & 0.000 \\
\hline NISSAN & 15762.00 & 22.854 & 522.324 & 9529.00 & 23.580 & 555.999 \\
\hline PEUGEOT & 192.00 & 0.278 & 0.078 & 547.00 & 1.354 & 1.832 \\
\hline RENAULT & 511.00 & 0.741 & 0.549 & 1422.00 & 3.519 & 12.382 \\
\hline SMART & 120.00 & 0.174 & 0.030 & 0.00 & 0.000 & 0.000 \\
\hline SUBARU & 14.00 & 0.020 & 0.000 & 88.00 & 0.218 & 0.047 \\
\hline SUZUKI & 1212.00 & 1.757 & 3.088 & 557.00 & 1.378 & 1.900 \\
\hline TOYOTA & 4183.00 & 6.065 & 36.787 & 3969.00 & 9.821 & 96.459 \\
\hline VOLKSWAGEN & 12819.00 & 18.587 & 345.482 & 2408.00 & 5.959 & 35.505 \\
\hline Total & 68967.00 & 100 & 1346.625 & 40412.00 & 100 & 1141.632 \\
\hline $\mathrm{Cr} 4$ & & 66.04 & & $\mathrm{Cr} 4$ & 54.37 & \\
\hline
\end{tabular}

Fuente: Elaboración propia con datos de la AMIA (2018). 
Tabla 2

VENTAS Y CONCENTRACIÓN DE LA INDUSTRIA AUTOMOTRIZ EN CANTIDADES (FEBRERO DE 2018)

\begin{tabular}{ccc}
\hline Ventas & Unidades & Cuota \% \\
\hline Subcompactos & $\mathbf{7 2 2 4 5}$ & $\mathbf{3 3 . 0 4}$ \\
\hline Compactos & 49213 & $\mathbf{2 2 . 5 1}$ \\
\hline De Lujo & 13514 & 6.18 \\
\hline Deportivos & 1292 & 0.59 \\
\hline SUV & 53123 & $\mathbf{2 4 . 3 0}$ \\
\hline Camiones Ligeros & 29006 & 13.27 \\
\hline Camiones pesados & 236 & 0.11 \\
\hline Total & 218629 & 100.00 \\
\hline CR3 (subcompactos, compactos y SUV) & & 79.85 \\
\hline
\end{tabular}

Fuente: Elaboración propia con datos de la AMIA (2018).

Tabla 3

NATURALEZA VARIABLES

\begin{tabular}{cccccc}
\hline Variables & Categoría & Transformación & Variables & Categoría & Transformación \\
\hline $\begin{array}{c}\text { Competidores } \\
\text { Precio }\end{array}$ & Numérica & Logaritmo & Subcompacto & Dummy & (1) Si es (0) No \\
\hline Poder mercado & Numérica & Logaritmo & Compacto & Dummy & (1) Si es (0) No \\
\hline Alto equipamiento & Dummy & (1) Si es (0) No & Sedan de lujo & Dummy & (1) Si es (0) No \\
\hline Medio equipamiento & Dummy & (1) Si es (0) No & Deportivo & Dummy & (1) Si es (0) No \\
\hline Austero & Dummy & (1) Si es (0) No & & (1) Si es (0) No \\
\hline
\end{tabular}

Fuente: Elaboración propia. 